\title{
A criterion of strong density of operator subalgebras
}

\author{
Antoni Wawrzyńczyk
}

\begin{abstract}
The purpose of the note is to show the usefulness of a simple criterion of density of a subalgebra $\mathcal{A} \subset L(X)$ in the strong operator topology for arbitrary real or complex locally convex vector space $X$. After proving the criterion we observe its efficiency obtaining short proofs of three important known density theorems plus a new one.
\end{abstract}

\section{Introduction}

Let $X$ be a locally convex real or complex vector space. Denote by $L(X)$ the space of linear continuous operators on $X$.

The strong operator topology on $L(X)$ is the topology of pointwise convergence of nets. A base of the neighbourhoods of zero in this topology can be parametrized by systems $\left(\mathcal{V}, x_{1}, \ldots, x_{k}\right)$, where $\mathcal{V}$ is a convex neighbourhood of zero in $X$ and $x_{1}, \ldots, x_{k} \in X$. It is given by the collection of sets

$$
\mathcal{U}\left(\mathcal{V}, x_{1}, \ldots, x_{k}\right)=\left\{A \in L(X): A x_{1}, \ldots, A x_{k} \in \mathcal{V}\right\}
$$

An operator $A \in L(X)$ belongs to the closure of a subset $\mathcal{S} \subset L(X)$ in the strong operator theory (denoted $\overline{\mathcal{S}}$ ) if and only if it can be approximated by elements of $\mathcal{S}$ on every finite-dimensional subspace of $X$.

Let $\mathcal{A}$ be a subalgebra of $L(X)$. We say that $x \in X$ is cyclic for $\mathcal{A}$ if $\mathcal{A} x=\{A x$ : $A \in \mathcal{A}\}$ is dense in $X$. The action of $\mathcal{A}$ is irreducible if every non-zero $x \in X$ is cyclic for $\mathcal{A}$.

Received by the editors in December 2015 - In revised form in March 2016.

Communicated by A. Valette.

2010 Mathematics Subject Classification : Primary: 47A15, Secondary: 47A65, 47A58.

Key words and phrases : Locally convex space, continuous operator, strong operator topology. 
Let us provide the dual space $X^{\prime}$ with the weak* - topology. Denote by $\mathcal{A}^{*}$ the subalgebra of $L\left(X^{\prime}\right)$ of all operators adjoint to the elements of $\mathcal{A}$.

For $v \in X$ and $\varphi \in X^{\prime}$ we denote by $\varphi \otimes v$ the rank one operator defined by $\varphi \otimes v(x)=\varphi(x) v$. Every rank one operator in $X$ is of this form.

The principal result of the paper (Theorem 2.1) states that $\overline{\mathcal{A}}=L(X)$ if and only if there exist $v \in X$ and $\varphi \in X^{\prime}$ which are cyclic for $\mathcal{A}$ and $\mathcal{A}^{*}$, respectively and $\varphi \otimes v \in \overline{\mathcal{A}}$.

In particular, if the action of $\mathcal{A}$ is irreducible and $\mathcal{A}$ contains a rank one operator, it follows that $\mathcal{A}$ is dense in $L(X)$.

In section 3 we obtain very simple proofs of density theorems known from the papers [Z], [MZ], [BS].

\section{The density theorems}

Let $X$ be a locally convex vector space.

Theorem 2.1. Let $\mathcal{A}$ be a subalgebra of $L(X)$. Suppose that $v \in X$ is cyclic for $\mathcal{A}$ and $\varphi \in X^{\prime}$ is cyclic for $\mathcal{A}^{*}$. If $\varphi \otimes v \in \overline{\mathcal{A}}$ then $\overline{\mathcal{A}}=L(X)$.

Proof. Fix $u \in X, f \in X^{\prime}$ and choose a convex neighbourhood of zero $\mathcal{V}$ in $X$. For arbitrary $k$ - tuple $x_{1}, \ldots, x_{k} \in X$ let $M=\max _{1 \leq i \leq k} \mid f\left(x_{i} \mid\right.$.

There is $T \in \mathcal{A}$ such that $u-T v \in \mathcal{V} / 2 M$ because $v$ is cyclic for $\mathcal{A}$. Choose $\varepsilon>0$ small enough to satisfy $\varepsilon T v \in \mathcal{V} / 2$.

The form $\varphi$ is cyclic for $\mathcal{A}^{*}$, hence there is $S \in \mathcal{A}$ such that

$$
\left|S^{*} \varphi\left(x_{i}\right)-f\left(x_{i}\right)\right|=\left|\varphi\left(S x_{i}\right)-f\left(x_{i}\right)\right|<\varepsilon, i=1, \ldots, k .
$$

Then:

$$
f\left(x_{i}\right) u-\varphi\left(S x_{i}\right) T v=f\left(x_{i}\right)(u-T v)+\left(f\left(x_{i}\right)-\varphi\left(S x_{i}\right)\right) T v .
$$

By the choice of the objects, each term in the sum belongs to $\mathcal{V} / 2$, hence $f\left(x_{i}\right) u-\varphi\left(S x_{i}\right) T v \in \mathcal{V}$.

For arbitrary rank one operator $f \otimes u$ and arbitrary basic neighbourhood of zero $\mathcal{U}\left(\mathcal{V}, x_{1}, \ldots, x_{k}\right)$ in $L(X)$ we have found $S, T \in \mathcal{A}$ such that

$$
f \otimes u-T(\varphi \otimes v) S \in \mathcal{U}\left(\mathcal{V}, x_{1}, \ldots, x_{k}\right) .
$$

Since $\varphi \otimes v \in \overline{\mathcal{A}}$ by assumption, we obtain that $f \otimes u \in \overline{\mathcal{A}}$. The algebra $\overline{\mathcal{A}}$ contains all rank one operators, so it is equal to $L(X)$.

Corollary 2.2. Suppose that the action of a subalgebra $\mathcal{A} \subset L(X)$ on $X$ is irreducible. Then $\mathcal{A}$ is strongly dense in $L(X)$ if and only if $\overline{\mathcal{A}}$ contains a rank one operator.

Proof. If the action of $\mathcal{A}$ on $X$ is irreducible then the action of $\mathcal{A}^{*}$ on $X^{\prime}$ is irreducible as well. Every non-zero vector $v \in X$ is cyclic for $\mathcal{A}$ and every non-zero functional $\varphi \in X^{\prime}$ is cyclic for $\mathcal{A}^{*}$. The proof follows by Theorem 2.1.

Corollary 2.3. Let $H$ be a Hilbert space and let $\mathcal{A} \subset L(H)$ be a self-adjoint subalgebra. If the action of $\mathcal{A}$ on $H$ is cyclic and the orthogonal projection on the 1-dimensional space spanned by the cyclic vector belongs to $\overline{\mathcal{A}}$, then $\overline{\mathcal{A}}=L(X)$. 


\section{Applications}

We present the simplified proofs of three important results concerning the generation of the space $L(X)$. We use the author's constructions of subalgebras $\mathcal{A} \subset L(X)$ and then we apply Theorem 2.1 or Corollary 2.2 for proving the density of $\mathcal{A}$ in $L(X)$.

1. Generation of $L(H)$ by two commuting $C^{*}$ - subalgebras.

Let $(H,(\cdot \mid \cdot))$ be a complex Hilbert space. In the paper [BS] the authors construct two $C^{*}$-commutative subalgebras of the space $L(H)$ which generate $L(H)$ in the strong operator topology. The construction uses a simple but very interesting fact that an arbitrary set $Z$ admits a structure of an abelian group. If $\left\{e_{i}\right\}_{i \in Z}$ is an orthonormal basis in $H$ and $Z$ is provided with the structure of an Abelian group, we can define a family of shift operators in $H$ by the formula

$$
S_{i}\left(e_{j}\right)=e_{i+j}
$$

The algebra $\mathcal{A}_{1}$ generated by the operators $S_{i} i \in Z$ is self-adjoint.

Let $P_{0}(x)=\left(x \mid e_{0}\right) e_{0}$, where 0 means the neutral element of the group $Z$. The operator $P_{0}$ is the orthogonal projection on the line generated by $e_{0}$. The space $\mathcal{A}_{2}=\mathbb{C} P_{0}$ is a 1-dimensional self-adjoint subalgebra of $L(H)$.

The main result of [BS] states that the subalgebra $\mathcal{A}$ generated by $\mathcal{A}_{1}$ and $\mathcal{A}_{2}$ is dense in $L(H)$ in the strong operator topology.

It follows immediately by Corollary 2.3.

2. $L(X)$ is strongly generated by two subalgebras of square zero.

An algebra $\mathcal{A}$ is of square zero if $A B=0$ for every $A, B \in \mathcal{A}$. In [Z] it was proved that for every Banach space $X$ there exist two subalgebras $\mathcal{A}_{i}, i=1,2$ with square zero which generate $L(X)$. The construction applied works perfectly for all locally convex spaces, so we prove the theorem supposing that $X$ is a real or complex locally vector space. We denote by $\mathbb{K}$ the field $\mathbb{R}$ or $\mathbb{C}$.

Let us fix $x_{0} \in X$ and $f_{0} \in X^{\prime}$ in such a way that $f_{0}\left(x_{0}\right)=1$.

Define two algebras consisting of rank one operators:

$\mathcal{A}_{1}=\left\{f \otimes x_{0}: f \in X^{\prime}, f\left(x_{0}\right)=0\right\}$ and $\mathcal{A}_{2}=\left\{f_{0} \otimes z: z \in X, f_{0}(z)=0\right\}$. A simple calculus shows that $\mathcal{A}_{i}, i=1,2$ are subalgebras with zero square. Let $\mathcal{A}$ be the algebra generated by elements of $\mathcal{A}_{1}$ and $\mathcal{A}_{2}$. By Corollary 2.2 we only need to prove that the action of $\mathcal{A}$ on $X$ is (algebraically) irreducible.

The space $X$ decomposes in the direct sum $X=\operatorname{ker} f_{0}+\mathbb{K} x_{0}$. Let $x \notin \operatorname{ker} f_{0}$. Then $\left(f \otimes x_{0}+f_{0} \otimes z\right) x=f(x) x_{0}+f_{0}(x) z$. For arbitrary $v=\lambda x_{0}+z \in X$, $z \in \operatorname{ker} f_{0}$ we can find $f \in X^{\prime}$ annihilating $x_{0}$ and such that $f(x)=\lambda$. Then $v=\left(f \otimes x_{0}+f_{0} \otimes\left(\frac{z}{f_{0}(x)}\right)\right)(x)$ belongs to the orbit of $x$ under $\mathcal{A}$. In particular the orbit of $x_{0}$ under $\mathcal{A}$ coincides with $X$.

For $x \in X$ such that $f_{0}(x)=0$ we apply $g \otimes x_{0}$ where $g\left(x_{0}\right)=0$ and $g(x) \neq 0$ obtaining $g \otimes x_{0}(x)=g(x) x_{0}$ and next we proceed as above.

The algebra $\mathcal{A}$ is strongly dense in $L(X)$ by Corollary 2.2.

3. For separable Banach space $X$ the algebra $L(X)$ is strongly generated by two of its elements. 
In the paper [MZ] V. Müller and W. Żelazko proved that for arbitrary separable Banach space there exist two elements of $L(X)$ which generate this algebra.

The construction of the corresponding operators is based on a result of Ovsepian and Pełczyński on the total bounded biorthogonal systems in separable Banach spaces.

Theorem 3.1. $[\mathrm{OP}],[\mathrm{P}]$ Let $X$ be a separable Banach space. There is a sequence $\left(x_{i}\right)$ in $X$ and a sequence $\left(f_{i}\right)$ in $X^{\prime}$ such that

1. $f_{m}\left(x_{n}\right)=\delta_{m, n}$ (the Kronecker symbol) $m, n \in \mathbb{N}$.

2. The linear span of $\left(x_{i}\right)$ is dense in $X$ in the norm topology.

3. The linear span of $\left(f_{i}\right)$ is dense in $X^{\prime}$ in the weak* - topology.

4. $\left\|x_{i}\right\|=1$, and $\left\|f_{i}\right\|<2$ for all $i$.

The conditions (1)-(4) assure that the operators

$$
R=\sum_{i=1}^{\infty} 2^{-i}\left(f_{i} \otimes x_{i+1}\right) \quad \text { and } \quad S=\sum_{i=1}^{\infty} 2^{-i}\left(f_{i+1} \otimes x_{i}\right)
$$

are well defined and bounded on $X$.

Since $R x_{i}=2^{-i} x_{i+1}$, the property (2) implies that the vector $x_{1}$ is cyclic for the subalgebra generated by the operator $R$.

Notice that $S^{*}=\sum_{i=1}^{\infty} 2^{-i}\left(x_{i} \otimes f_{i+1}\right)$, where we identify $x_{i}$ with the functional on $X^{\prime}$ defined by $\varphi \rightarrow \varphi\left(x_{i}\right)$. Indeed,

$$
\begin{aligned}
S^{*}(\varphi)(x) & =\varphi\left(\sum_{i=1}^{\infty} 2^{-i} f_{i+1}(x) x_{i}\right)=\sum_{i=1}^{\infty} 2^{-i} f_{i+1}(x) \varphi\left(x_{i}\right) \\
& =\sum_{i=1}^{\infty} 2^{-i}\left(x_{i} \otimes f_{i+1}\right)(\varphi)(x)
\end{aligned}
$$

In particular we obtain $S^{*}\left(f_{i}\right)=2^{-i} f_{i+1}$. By (3) the functional $f_{1}$ is cyclic for the algebra spanned by powers of the operator $S^{*}$.

By direct computation we get the formula $4 S R-R S=f_{1} \otimes x_{1}$. The assumptions of Theorem 2.1 are satisfied, hence the algebra generated by the operators $R, S$ is dense in $L(X)$.

4. If a locally convex space $X$ admits an operator without closed invariant subspaces then $L(X)$ is strongly generated by two elements.

In the previous subsection it was proved that for separable Banach space $X$ the algebra $L(X)$ is generated by two of its elements. The same fact holds for several special spaces without the assumption of the separability.

Suppose that in a locally convex space $X$ there exists $T \in L(X)$ which has no closed invariant subspace. The action of the algebra of polynomials $P(T)$ of this operator is irreducible on $X$. If $S$ is an arbitrary rank one operator on $X$ then by Corollary 2.2 the operators $T$ and $S$ generate $L(X)$. 


\section{References}

[BS] R. Berntzen, A. Sołtysiak, On strong generation of $B(\mathcal{H})$ by two commutative $C^{*}$-algebras, Studia Math. 125 (2) (1997), 175-178.

[MZ] V. Müller, W. Żelazko, $B(X)$ is generated in strong operator topology by two of its elements, Czech. Math. Journ., 39 (114) (1989) 486-489.

[OP] R. I. Ovsiepan, A. Pełczyński, Existence of a fundamental total and bounded biorthogonal sequence, Studia Math. 54 (1975), 149-159.

[P] A. Pełczyński, All separable Banach spaces admit for every $\varepsilon>0$ fundamental total and bounded by $1+\varepsilon$ biorthogonal sequences, Studia Math. 55 (1976), 295-304.

[Z] W. Żelazko, $B(X)$ is generated in strong operator topology by two subalgebras with square zero, Proc. R. Ir. Acad., 88 A (1) (1988) 19-21.

Departamento de Matemáticas,

Universidad Autónoma Metropolitana-Iztapalapa AP 55-534,

09340 México D. F., México

email:awaw@xanum.uam.mx 